\title{
Evidence Based Military Medicine - The NATO Trauma Registry Initiative
}

\author{
BALÁZS Róbert ${ }^{1}$, KOPCSÓ István²
}

\begin{abstract}
For thousands of years Medicine was practiced in empiric and authoritarian ways. Physicians and other medical personnel always treated sick people with the principle of "nil nocere", meaning "do no harm", but the procedures sometimes either were not effective or did cause harm. Modern scientific methods in medicine enabled the scientists to provide firm results and evidence of a particular treatment or procedure in health care provisions.

In a controlled clinical environment the prospective, double blinded, multi-centric, randomized trial became the golden standard of research, because this method provided the most solid basis of testing a hypothesis.

In Military Medicine the operational environment and battle rhythm define the framework for the practice. It is impossible to design a trial with all the aforementioned requirements in battlefield settings, however small scope prospective trauma care studies are now getting approval and some of them have been already published. The tools for this research are the national Military Trauma Registry Systems, which are available now in a few countries. The NATO Trauma Registry Initiative is a multinational effort for the exchange of operational trauma care data among the NATO Military Medical Services, to foster the improvement of Military Medicine and to provide more solid evidence for treatment.
\end{abstract}

\section{Foreword}

The chronology of the NATO Trauma Registry (NTR) takes one back in time to 2008. The successes of the few national military trauma registries became apparent and the Chiefs of Military Medical Services in NATO (COMEDS) requested a study from the NATO Research and Technology Organization $^{3}$ (RTO). The RTO described the national trauma registries and released a firm recommendation and the summary of the benefits of a common database. This technical report was published in March 2008. [1] The report provided the reference point for the development of the NTR.

\section{Executive Summary}

"One of the precepts of military medical support is to constantly maintain and improve the quality of healthcare which is available to our deployed personnel. When there is a political

1 NATO Support Agency Capellen, Luxembourg, e-mail: robert.balazs@nspa.nato.int

2 Supreme Headquarters Allied Powers Europe (SHAPE) Mons, Belgium

3 New name is NATO Science and Technology Organization (STO) 
decision to put troops in harm's way, there is a concomitant duty to implement processes that will improve quality of care in an institutionalised way. One tool which has been found useful in this context by several nations is that of a trauma registry." [1]

Continuous improvement of health care in operations needs to be supported by various analyses of injuries sustained in combat situations. The underlying data should be captured, stored and utilised for comprehensive research and performance improvement.

Although the imperative of such a tool is admitted, there are only few nations employing trauma registries on a national level. There is a particular limitation for nations whose casualty numbers and case loads are not sufficiently large to allow scientifically and statistically robust analysis. Moreover, there is no standardized means of communication or exchange of information. This factor limits the systematic exploitation of lessons learned.

The NATO Trauma Registry Task Force (NTR TF) was established in late 2010 to accelerate the process of the implementation of a common Trauma Registry in NATO. COMEDS endorsed the initiative and mandated the Task Force to validate the progress by using a pilot study [2].

\section{Methodology}

The pilot study assessed the feasibility of the NTR. It is a descriptive study. Besides the analysis of virtual patient data MILMED COE performed field health data collection in the Area of Responsibility of the International Stabilisation and Assistance Forces (ISAF). The field deployment included one week data collection in Kabul FRA Role3 hospital and one week in Mazar-e-Sharif DEU Role3 hospital. From 15-30 JAN 2012 two MILMED COE medical staff officers, who have been previously trained in US trauma system, were deployed to execute the task.

During the pilot study the NTR Core Data Elements (defined by different COMEDS Working Groups and Expert Panels), US JTTR v3.2 Data Collection Form and UK Trauma Audit Form v5.1 were utilized. ${ }^{4}$ The team collected anonymous patient data according to the data handling policy. The collected data is classified as UNCLASSIFIED.

The team mission was affected by one major limiting factor:

- The theatre weather conditions limited the data collection in FRA Role3 hospital to three days.

Parallel with the planning and performance of the field data collection, the TF member NATO Consultation, Command and Control Agency (NC3A) constructed the experimental NTR import interface. NC3A tested the NTR-US-UK exchange capabilities using the NATO Trauma Registry Core Data Elements. The technical report from the Agency will be discussed in this report concisely.

\section{Major conclusions}

Having proven the technical feasibility of communication and data exchange between different registries further standardization of data elements and development of related processes are needed.

4 All three data collection forms are available from the authors. 


\section{Key recommendations}

- Development of a standardized data dictionary (in accordance with STANAG 2231)

- Engagement of the TF in further development from "Registry" to "System" during the initiated commercial development phase of the NTR

\section{Introduction}

The NATO Trauma Registry (NTR) is a military medical tool that will link participating nations to improve operational medical awareness, monitor casualty care, share experience and analysis of trauma management among nations and so ensure that state-of-the art trauma care is provided. The principle purpose of a NTR is to act as a quality assurance system for the management of military medical trauma from point of injury to rehabilitation. It is a tool to support continuous detailed clinical audit and research and improve the provision of care for frontline personnel.

The NTR project was initially outlined by the NATO Research and Technology Organisation (RTO). Human Factors \& Medicine (HFM) was tasked to analyse the current national trauma registries and make proposals to implement such a tool NATO-wide. RTO predicted the following potential benefits:

- Aid in the classification of injuries sustained in military operations

- Track casualty outcomes and impairment

- Develop standardized clinical treatment guidelines

- Ensure effective process monitoring

- Improve the development and use of protective equipment and vehicle protection

- Inform and focus medical research

- Assess and improve treatment quality[1]

It is crucial that the final RTO report recommended establishing a common NATO trauma registry.

The Committee of the Chiefs of the Military Medical Services (COMEDS) endorsed the project, tasking the Military Health Care WG (MHC WG) and the Medical Command Information Systems Expert Panel (MedCIS EP) to work on its development. Later MHC WG involved the Emergency Medical Expert Panel (EM EP) to provide its expertise and help to define the NTR core data elements. The Medical Communication and Information Systems Expert Panel (MedCIS EP) contributed STANAG 2543 promulgated in 2009 and STANAG study draft 2231, but this was later withdrawn from the ratification process. 


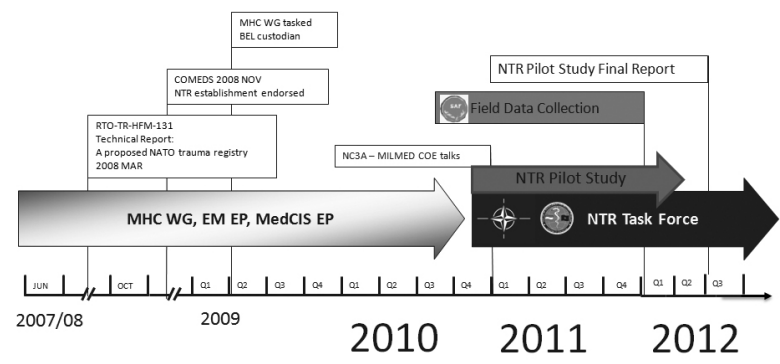

Figure 1. NATO Trauma Registry timeline $e^{5}$

In 2010, acknowledging the comprehensive work done by the custodian, the NATO Trauma Registry project was reaffirmed. A NTR Task Force (TF) - upon receiving mandate from COMEDS - was established under the umbrella of MHC WG, with MILMED COE as coordinating secretary supported by volunteer participating nations (BEL, CZE, DEU, FRA, GBR, NLD, USA) and Allied Command Operations (ACO). NC3A was tasked by Allied Command Transformation (ACT) to support the project and facilitate the project development [3]. The TF got the mandate to accelerate the process and validate the progress using a pilot study. The timeline of the TF activities are shown in Fig. 2.

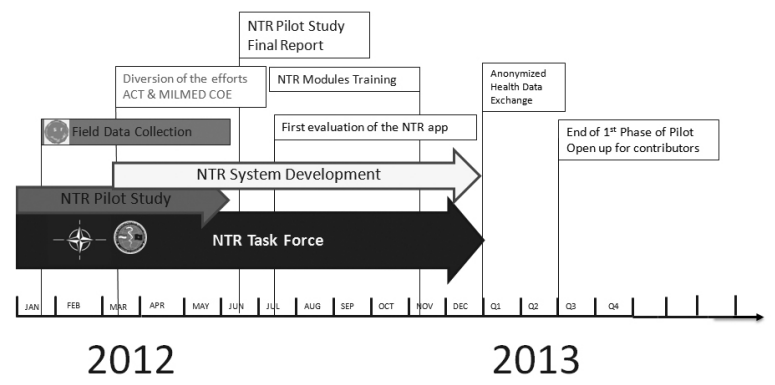

Figure 2. NATO Trauma Registry Task Force Timeline ${ }^{6}$

\section{Problem Statement}

A number of nations are employing trauma registries but they are doing it on a limited national level. There are no standardized means of communication or exchange of information amongst the involved nations and this fact limits the opportunities to learn quickly from the experience of others and prohibit a broader analysis of trauma to support best medical practise in military deployments. This is a particular limitation for nations whose casualty numbers and case loads are not sufficiently large to allow scientifically and statistically robust analysis. The result is that only anecdotal information is available about the current combat trauma care of the casualties.

In order to facilitate and structure the further development of a common NTR, with the benefits for all contributing nations, the TF has designed this pilot study.[2]

5 Author's own figure created in 2012, presented in Trauma Innovation Conference in London UK

6 Author's own figure created in 2013, presented in Trauma Innovation Conference in London UK 


\section{Pilot objectives}

\section{Goals/Overall Objectives:}

1. To assess the feasibility of the NTR.

2. To assess that the validity / significance of military medical research is higher in multinational than in national approaches.

3. To determine that evidence in military medical research can be achieved when it is based on a qualitatively and quantitatively sufficient set of standardized data. ${ }^{7}$

\section{Purpose:}

1. To develop a working model of NTR; to merge the numbers of national cases into a single database and henceforth provide a scientifically robust pool of information for studies and analysis.

2. To encourage nations to exchange and share anonymous data.

\section{Output}

1. Established functional interface among national trauma registries

2. Communication strategy among NATO TF Nations.

\section{Activities}

1. To identify a limited number of parameters for a descriptive study design based on NATO TRAUMA REGISTRY CORE DATA ELEMENTS [10]

2. To identify participating nations and their current systems (internal system model compared to NTR model)

3. To utilize the UK/US JTTR as a template of national trauma registries

4. To develop a Trauma Registry System Data Model in order to exploit the trauma registry data

5. To develop an NTR Data Exchange Model to enable exchange of core data elements

6. To utilize algorithm for Global NTR ID in order to avoid data redundancy

7. To design the preliminary rules and procedures to support the NTR model

8. To do a test run of import / export based on available systems

9. To define communication media and periodicity for the data exchange (CD, mail, attachments, etc.)

\section{Methodology}

\section{Type of study:}

This pilot study is designed to assess the feasibility of the NTR. It's a descriptive study.

\section{Inclusion / exclusion criteria:}

NTR inclusion criteria are defined in Annex B of RTO TR-HFM-131: all patients (military and civilian) with physical trauma seen at Role 2 or above requiring one of the following [1]:

- Surgical procedure/treatment

- Activation of the trauma team, partial or full.

- Admittance in MTF

7 Goal 2 and 3 require further research and will be discussed elsewhere. 
- Transport to a higher level

- All deaths

\section{Data collection}

Data collection and "Minimum Data Set” are defined in Annex C of RTO TR-HFM-131.

After the data collection phase, national data (AFG, DEU, FRA, GBR and USA) were imported into the NTR Database.

\section{Evaluation Criteria $^{8}$}

- Quantity of anonymous trauma data

- Functionality of communication among systems

- Timeliness

- Centralised control and handling

- Accessibility

- Collection method

- Effectiveness and efficiency

- Quality and standards

- Usability of collected data for lessons identified

- Further need of data standardization (e.g. ICD-10) or any interoperable standardized nomenclature according to STANAG 2231.

- User-friendliness of the manual and the requirement for specific training for data collection and registering.

\section{Study Results / Observations}

\section{Results of the field experiment}

The field data collection aimed to populate trauma patient data available in theatre military treatment facilities (MTF). The two weeks period of deployment was split into two parts. In the first week the team visited the FRA Role3 hospital in Kabul International Airport (KAIA), the second week was spent in the DEU Role3 hospital in Mazar-e-Sharif (MeS), ISAF Regional Command North [5].

\section{Description of activities}

The COE team met the MEDAD of RC North that included discussion of current epidemiological trends and preventive medical trends.

The team received a hospital familiarization brief and situational awareness presentation in the facilities. The multinational medical team in both facilities were aware of the NTR project. Trauma patients were selected with the active assistance of the hospital leadership according the NTR inclusion criteria.

8 Summary of Evaluation criteria results in Annex A 
In Kabul the FRA hospital included BGR, CZE, HUN and USA personnel. The patient documentation was mainly in English; FRA military casualties sometimes were documented only in French. Our team was affected by the French hospital rotation and transfer of command in the particular week. The number of days spent in Kabul was reduced due to the weather and the consequent flight restrictions.

In Mazar-e-Sharif the hospital consisted of DEU, HUN and USA medical personnel. The patient documentation was in English and German, for non-DEU patients the medical record was always in English. The DEU hospital provided office space and a standalone laptop with fixed IP (internet protocol) address.

The team tested data availability in coherence with the NATO Trauma Registry Core Data Elements, and the UK Trauma Audit Form v5.1, US JTTR v3.2 Data Collection Form. These forms were available electronically as MS Word documents except the US form, which was in Adobe Portable Document Form (PDF). The US dataset was employed on printed paper forms.

\section{NTR Core data elements}

NTR Core Data Elements are based on NATO RTO HFM-131 report recommendations. COMEDS tasked the Military Health Care Working Group (MHC WG) to provide minimum requirements for collectible trauma data. In the MILMED COE field data experiment the data set was utilized and recommendations are the following:

1. Some of the fields are not collectible from current medical documentation.

2. Some data fields affect national sensitivity e.g. weather conditions, vehicle and personal protection classification, definite geographic location of the incident/accident.

\section{UK Trauma Audit Form v5.1}

The UK employs a comprehensive joint trauma theatre system. The trauma registry is the backbone of the system providing a significant pool of data for patient care and also for personal protection improvements. UK JTTR is managed and maintained by the Defence Analytical Services and Advice (DASA), this organization supports scientific research and provides advice to different military levels.

In the operations the registrar is embedded in the clinical staff, those patients who meet the inclusion criteria are registered in the Trauma Audit Form (TAF). This is an MS Word document template where the entry fields are only open for editing and formatting. Drop down menus are provided in most of the entry fields.[7]

The TAF form is emailed when completed, and the data import to the registry is performed by the Academic Department of Military Emergency Medicine (ADMEM), in Birmingham.

GBR kindly distributed the latest version of the JTTR to MILMED COE and other nations on a bilateral basis. COE is entitled to install and experiment with the application. The team did not get training on the system, however the user manual provided with the UK JTTR application was sufficient.

In the MILMED COE field data experiment the data set was utilized and observations are the following:

1. All data entry fields have instructions/dictionary 
2. The TAF represents the UK national perception of casualty care flow. There are limited options for deviation if the patient care is performed by other national standards (e.g. not merging of Role1 and Pre-hospital).

3. Performance indicators are embedded in the form and reflect the most important quality control elements.

4. Coding and classification is done by the registrar, wounds are classified according the Red Cross standards. Trauma scoring codes (AIS, ISS, NISS, RTS, TRISS, and AS$\mathrm{COT}$ ) are calculated also by the registrar. In the form the diagnosis is narrative and not coded to WHO ICD or SNOMED.

5. DASA are coding the diagnosis to ICD 10 and OPCS 4 during the data procession.

\section{US JTTR v3.2 Data collection form}

The Joint Trauma System (JTS) is the Department of Defense global trauma system. The Joint Theatre Trauma System (JTTS) is the deployed team into the operational Area of Responsibility (AOR).

The Department of Defense Trauma Registry (DoDTR), is the combination of the Storeand-Forward and web-based Joint Theater Trauma Registry (JTTR) and they are the backbone of JTS [6]. Main objectives of the Trauma Registry (JTTR) are to support trauma care development and to facilitate performance improvement.

US DoDTR is maintained to provide accurate data for clinical practical guidelines, for prospective and retrospective studies and it provides important input for medical planning.

The MILMED COE team was trained on US DoDTR in a three weeks programme, together with other Trauma Nurse Coordinators (TNC) as part of the regular pre-deployment training of the JTTS. The TNCs are dedicated to take care of the DoDTR and rotated on a six months basis.

The US kindly distributed a limited version of Store and Forward JTTR application for MILMED COE experimentation. A user manual was also provided.

In the MILMED COE field data experiment the US DoDTR data set was utilized and observations are the following (data collection form was printed out and used for data capture):

1. Records are facility orientated and focusing on Role3 hospital level. Optional Role2 / Forward Surgical Team data collection is under development.

2. US DoDTR has a strong coding capability; the application has embedded calculators transforming narrative text to different diagnosis codes and trauma scoring codes. The current disease classification is ICD-9. The US most likely skip the ICD-10 and will adopt ICD-11. The coding, diagnosis, procedures and severity of trauma allow statistical analysis and also association of different cases.

\section{Result of the NC3A data exchange experience}

NC3A $^{9}$ created a schema in Extensible Mark-up Language (XML) to enable the data exchange between the NTR prototype and the national registries. This schema was created in a specific type of XML called XSD.

9 NCIA is the new name of the agency 
This XSD schema is functioning as an interface ahead of the NTR database layer and controlling the data messages before integrating data into the NTR.

The NTR XSD schema was created according the Core Data Elements and was modified after the NTR TF Budapest meeting (mid-October 2011). Currently version 1.0 is published on the MILMED COE NTR portal.

Summary of results of data exchange episodes:

- US DoDTR

- NC3A received the same message (containing one trauma record) a number of times, and at the beginning of Feb 2012 we were able to successfully import this record into NTR. It helped prove the concept of the pilot study.

- UK JTTR

- At the end of January 2012 NC3A received a CD with data files, but this data is NOT compliant to the NTR schema, therefore we could not import it. Due to resource constraints, the UK could only have sent compliant data later.

- NC3A has been working on the NTR User's Operational Requirements (UOR) / SRS rather than on developing the NTR capability further; this is in line with ACT requirements.

- To develop the NTR capability further test imports and exports are essential to carry out.

- UOR work revealed that changes should be made to the schema.

\section{Discussion and Recommendations}

\section{Discussion of IT interoperability}

Data export and data message construction is a national task. The current and future NTR contributing nations have to adjust their data set in line with the minimum core data elements. Data export tools should be capable of extracting the minimum core data elements and sending them in a defined and standardized XML language.

The UK and US DoDTR were amended to include the minimum core data elements; nations are free not to share elements they find sensitive or classified by a national decision.

NTR Prototype I. needs further adjustments to enable more effective data exchange. Constraints of the XSD schema need to be opened, the current model data fields are restrictive and refuse non- fully compliant data transmission.

1. Example 1. If One element is sensitive for sharing and one nation sends no-data the system rejects all the message, there is no option to integrate partially

2. Example 2. XSD schema was able to receive only the literal text for the particular data field, if the text was altered by the sender the schema rejected the whole message and lost the other data field elements

The recommendation is to further develop the schema, “open the gates" and eliminate constrains. The NTR model/prototype II will enable the test patient data exchange while the received data will provide a pattern for data standardization and data dictionary development.

Upon refinement of the NTR model/prototype II the final model will enable the Task Force to perform real patient data exchange.

Fig. 3 and 4 depicts the proposed data flow and data management from point of data collection to final analysis. 


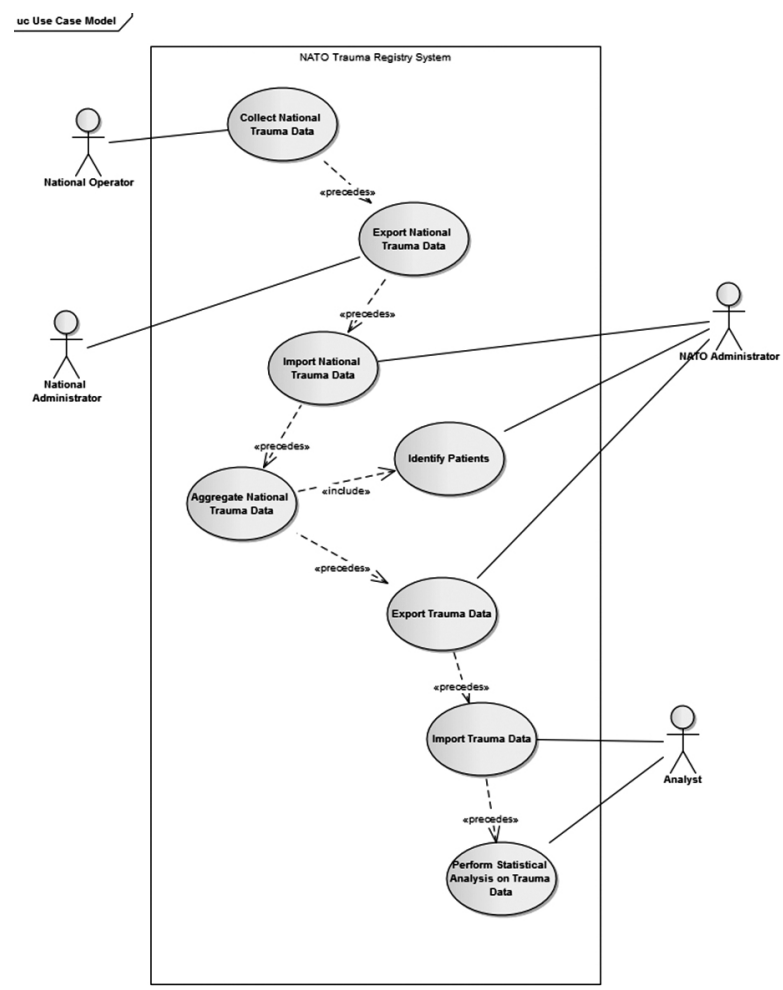

Figure 3. Data flow [11]

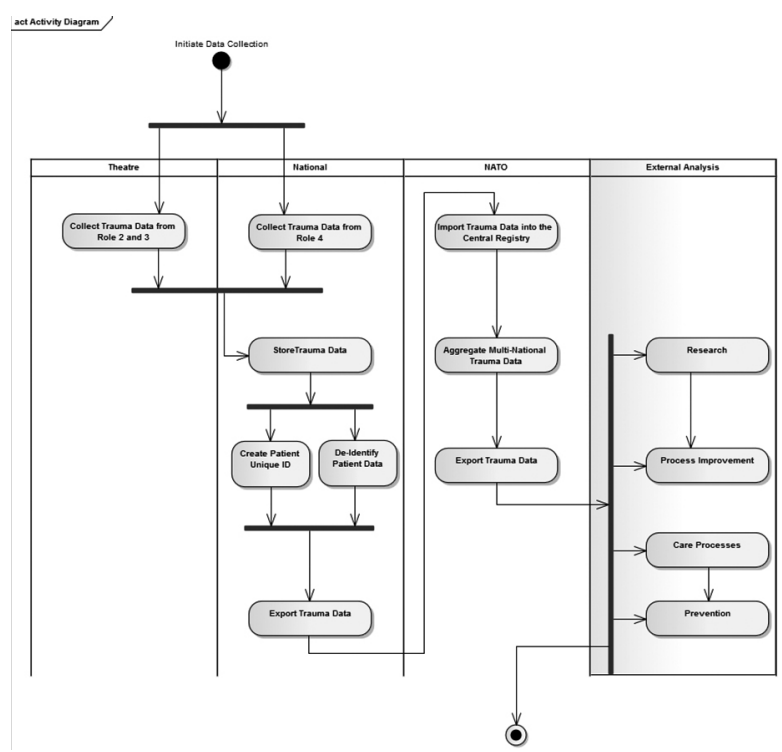

Figure 4. Proposed data management [11] 


\section{Discussion of data elements collection}

As a result of the individual data entries and the discussions with medical planners there were strong links identified where quality improvement in trauma care and epidemiological surveillance conjugate, and Force Health Protection approaches apply.

Due to the low sample size statistical analysis was not carried out on captured data. Efforts were focused on practical observations regarding data collection in a clinical environment where trauma registry is a novel tool. Additional findings were made from the parallel utilization of the three datasets.

Entering the same cases there was different achievable compatibility with the UK and US data form. As far as all three forms are concerned there are major variances in inclusion criteria, data sets and data dictionaries as well. The current main deficiency of the NATO Trauma Registry Data Elements is the lacking data dictionary and missing specifications of numerous entries.

The available registries focus on hospital care and do not fully capture pre-hospital data.

\section{Conclusion}

Medical solutions in extensive kinetic military operations in austere environments have been under continuous evolution. The evolving changes in casualty management policies require continuous adaptation of the injury surveillance techniques. It is important to ad flexibility to the different national and NATO injury surveillance systems, but in the meanwhile a core common standardization is necessary to ensure Completeness and Data Quality.

Further research recommended:

1. Data dictionary for all entry fields needs to be elaborated. It is advised to take over data definitions from the UK/USA trauma registry if available and feasible.

2. Explore NTR related Quality Improvement and Epidemiological Surveillance aspects and assess the links between Clinical Governance in Trauma Care and Force Health Protection.

3. Survey the NTR tactical level users' operational requirements and support the development of the System part. (collecting, analysis, reporting and dissemination)

4. Evaluate the deliverable products in terms of simplicity, flexibility, data quality, acceptability, representativeness, and timeliness.

If the quantity of data is sufficient, and further data standardization is performed statistical analysis (case control studies for example) should be realized in a second time approach to evaluate the impact of different factors on survival and general outcome.

\section{Description of the establishment of a new trauma system}

NTR development is in the phase when the major difficulties could be examined and dissolved through various trials and experiments in multinational environment. The NTR Pilot Study is now concluding the phase 1 (feasibility), but the Task Force has already proposed to maintain their future presence in order to continue the work in coordination, harmonization of the NTR, providing expertise for the nations, and providing assistance for standardization in the further (planning) process. (Fig. 5). 


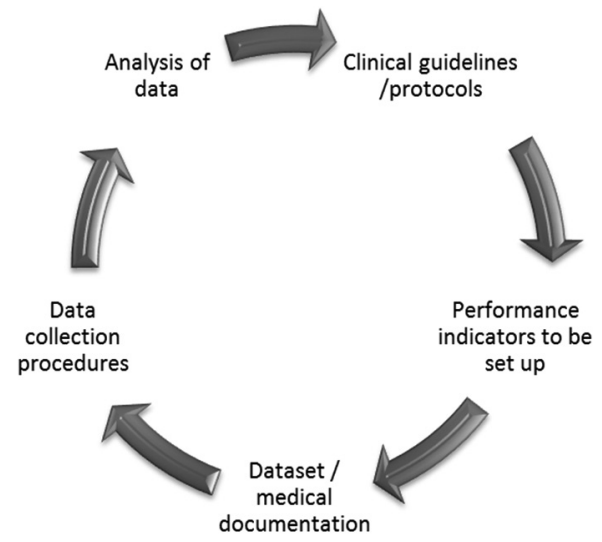

Figure 5. Proposed cycle of trauma system development ${ }^{10}$ [11]

The cycle depicts the continuous process and drive of the different elements in the trauma system development. Any of the five steps can be an entry point; a nation perhaps already has operational medical guidelines available, another nation only has some collected data, but has no analysis capability in place. Perhaps the simplest way is to start collecting available data, and later refine the clinical documentation and broaden the data collection matching the findings. When enough clinical data are available than clinical guidelines can be developed. 


\section{Annex A}

\section{Evaluation Criteria results}

- Quantity of anonymous trauma data

- According to data availability, in Multinational Medical Units there are a sufficient number of multinational casualties

- Functionality of communication among systems

- Available with the NTR interface

- Timeliness

- Can be evaluated after enabling smooth communication between individual registries not forgetting that NTR is not a "near real time tool"

- Centralised control and handling

- Depending on future set up and structure of a NTR

- Accessibility

- Requires standardization between individual registries and data sharing agreements

- Collecting method

- Dedicated personnel for collection is recommended

- Effectiveness and efficiency (e.g. integrated function or extra posts in theatre)

- To be evaluated ("system") later when the system is operational

- Quality and standards

- To be evaluated ("system”) later when the system is operational

- Usability of collected data for lessons identified

- To be evaluated ("system”) later when the system is operational

- Further need of data standardization (e.g. ICD-10) or any interoperable standardized nomenclature according STANAG 2231.

- To be initiated asap (next step)

- User-friendliness of the manual and the need for specific training for data collection and registering

- To be evaluated after the finalization of the data dictionary

\section{References}

[1] NATO RTO-HFM: Proposed NATO Trauma Registry - Final Report of Task Group HFM-131/RTGA. 2008.

[2] NTR Task Force: NATO Trauma Registry Pilot Study Proposal 2.2. 2011.

[3] Allied Command Transformation (ACT): Point Paper on NATO Trauma Registry Development. 2011.

[4] NTR Task Force: Minutes of NTR TF meetings. 2011

[5] MILMED COE: NATO Trauma Registry Pilot Study - Execution plan for field data collection in ISAF. 2012.

[6] Store and Forward (US) Joint Theater Trauma Registry v3.2 User Guide. 2011.

[7] UK Joint Traumatic Theatre Register User Manual v1.0. 2011.

[8] BALÁZS R.: NATO Trauma Registry - Evidence Based Military Medical Care for the Alliance. Medical Corps International Forum, 2 (2012), 52-54.

[9] BALÁZS R.: NATO Trauma Registry Phase 2 - Capability Development. Medical Corps International Forum, 2 (2013), 60-61.

[10] COMEDS Military Health Care Working Group meeting. November 2010.

[11] NATO Trauma Registry Pilot Final Report 2011. http://www.coemed.org/ntr/index.php/ntrfinal-report (downloaded: 21102013 